\title{
NeuroImage
}

ELSEVIER

www.elsevier.com/locate/ynimg

NeuroImage 30 (2006) 1069 - 1076

\section{Doing the right thing: A common neural circuit for appropriate violent or compassionate behavior}

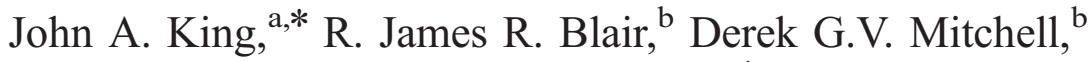 \\ Raymond J. Dolan, ${ }^{\mathrm{c}}$ and Neil Burgess ${ }^{\mathrm{a}, *}$ \\ anstitute of Cognitive Neuroscience and Department of Anatomy, University College London, 17 Queen Square, London WC1N 3AR, UK \\ ${ }^{\mathrm{b}}$ Mood and Anxiety Disorders Program, National Institute of Mental Health, National Institute of Health, Department of Health and Human Services, \\ 10 Center Drive, Bethesda, MD 20892-1381, USA \\ ${ }^{\mathrm{c}}$ Wellcome Department of Imaging Neuroscience, Institute of Neurology, University College London, 12 Queen Square, London WC1N 3BG, UK
}

Received 8 July 2005; revised 28 September 2005; accepted 5 October 2005

Available online 22 November 2005

\begin{abstract}
Humans have a considerable facility to adapt their behavior in a manner that is appropriate to social or societal context. A failure of this ability can lead to social exclusion and is a feature of disorders such as psychopathy and disruptive behavior disorder. We investigated the neural basis of this ability using a customized video game played by 12 healthy participants in an fMRI scanner. Two conditions involved extreme examples of context-appropriate action: shooting an aggressive humanoid assailant or healing a passive wounded person. Two control conditions involved carefully matched stimuli paired with inappropriate actions: shooting the person or healing the assailant. Surprisingly, the same circuit, including the amygdala and ventromedial prefrontal cortex, was activated when participants acted in a context-appropriate manner, whether being compassionate towards an injured conspecific or aggressive towards a violent assailant. The findings indicate a common system that guides behavioral expression appropriate to social or societal context irrespective of its aggressive or compassionate nature.
\end{abstract}

(c) 2005 Elsevier Inc. All rights reserved.

\section{Introduction}

A critical feature of human behavior is an ability to act in the manner appropriate to the current social or societal context. Failure to do so can result in social exclusion and characterizes disorders such as psychopathy. In this study, we use the context of a video game to examine the neural system mediating the generation of two different extremes of behavioral response (violence and compassion) that are appropriate in different contexts. By using

\footnotetext{
* Corresponding authors.

E-mail addresses: John.King@ucl.ac.uk (J.A. King), n.burgess@ucl.ac.uk (N. Burgess).

Available online on ScienceDirect (www.sciencedirect.com).
}

extreme forms of behavior, we hoped to maximize the signal for detection by an fMRI scanner. By including both violent and compassionate actions, we hoped to identify the neural systems involved in generating socially appropriate behavior in general, rather than those involved in behavior of one specific type.

In our task, participants entered virtual 3D rooms each of which contained either a passive casualty or an aggressive humanoid assailant. As they entered the room, they were equipped with either a gun or a bandage and were instructed to use the object on whatever was inside the room: to shoot or heal the assailant or casualty. The two experimental conditions involved appropriate behavior within the context of the video game: shooting the attacking humanoid or healing the passive casualty. The two control conditions involved carefully matched stimuli and actions combined inappropriately: shooting the casualty or healing the attacking humanoid (see Materials and methods). We thus sought to compare the neural response during appropriate violent and compassionate behavior to those during matched control conditions involving inappropriate behavior. We note that this framework imposes the necessary action for each trial rather than granting free-choice, and as such can be considered a case of intentional norm violation. This was a necessary manipulation as subjects would otherwise be expected to only perform what they considered appropriate actions.

The amygdala and orbitofrontal/ventromedial prefrontal cortex are of particular interest for our study, since lesions of these areas can disrupt the expression of socially appropriate behavior (Damasio, 1994; Grafman et al., 1996), and orbitofrontal cortex has been implicated in intentional norm violation (Berthoz et al., 2002). In addition, individuals with psychopathy, who present with reduced prosocial behavior (Hare, 1991) and with inappropriate use of violence, can present with amygdala and orbital frontal cortex dysfunction (Blair, 2003; Kiehl et al., 2001). The amygdala is strongly connected with medial and orbital prefrontal cortex as well as hippocampus and insular, temporal, and occipital cortices 
(Amaral and Price, 1984). To our knowledge, only one study has examined the neural bases of aggressive responses in healthy individuals: an fMRI study reporting frontal involvement in the expression of imagined aggressive behavior (Pietrini et al., 2000). Relatively little previous work has considered the neural systems mediating the opposite of aggression, i.e., compassionate behavior, or behavior undertaken with the goal of helping another individual. In the prisoner's dilemma game (Rilling et al., 2002), mutual cooperation is associated with activation in regions linked with reward processing: nucleus accumbens, the caudate nucleus, anterior cingulate, and ventromedial frontal/orbitofrontal cortex. However, this involves mutual aid rather than compassion, which is better exemplified by the Good Samaritan-like behavior of helping an unknown victim encountered on a single occasion. A recent study revealed the involvement of the anterior insula in empathy for the apparent suffering of loved-ones (Singer et al., 2004), but in that case, participants took no action, whereas here we are concerned with actively compassionate behaviour.

\section{Materials and methods}

\section{Participants}

Twelve healthy right-handed volunteers participated, six male and six female (mean age 22.4 years, range $18.7-38.2$ years). All participants gave informed written consent in accordance with requirements of the local medical ethics committee.

\section{Virtual environments}

The virtual environment was constructed using the Unreal engine developed by Epic Games Inc. Additions to the basic system were made using Microsoft Visual $\mathrm{C}++$ (to enable communication via the computer's parallel port) and 3D Studio Max (to make the bandage model animation). The environment consisted of a row of 120 identical square rooms connected by narrow passageways, with doors at each end of the passageways. Each room contained either a casualty or an aggressive humanoid and each passageway either a gun or a bandage.

\section{Training}

Before moving to the scanner, participants were familiarized with the environment and given practice trials. Familiarization involved learning to use the keyboard to navigate around the environment. In order not to dull the affective response to the experimental tasks, the practice tasks involved learning to use the gun to shoot a number of inert targets. Thus, the participants were unaware of the nature of the tasks they would be performing in the scanner. Their instructions were to enter each room, pick up the object they would find in the doorway, and use it to deal with the individual they encountered.

\section{Experimental tasks}

Each trial of the experiment involved the participant entering a virtual room and encountering either a male or female human (who slowly approaches) or a nonhuman assailant (all of these are hereafter referred to as the target). On entering the room, the participant received either a gun or a bandage. In the case of receiving a bandage, the target would initially be wounded (i.e., covered in blood). In the case of receiving a gun, the target would initially be healthy (i.e., no blood; see Fig. 1 for examples of the experimental conditions). This produced 4 types of condition involving: human + gun, human + bandage, nonhuman + gun, and nonhuman + bandage.

In all cases, the participant's task was to use the instrument to reverse the state of the target: the gun would be used to injure, and eventually kill, the initially healthy targets, while the bandage was used to cure the injured targets. These processes involved interactions based on the normal conventions of $3 \mathrm{D}$ video games - the participant could move around in the VE and use an action button to activate their instrument. For the gun, this caused it to fire (creating a flash, a gunshot sound, and, if successfully aimed, screams and an increase in blood on the target); for the bandage, it caused it to move over the target (resulting in a 'rustling cloth' sound and, if successfully aimed, screams and a decrease in blood on the target).

The auditory and visual stimuli in shooting and bandaging trials were balanced as much as possible in terms of the amount and nature of screams and the range and total amount of blood. The appearance of the target models was variably bloody according to their level of injury - thus when a shot hit an uninjured target, blood appeared, and when a bandage was applied, blood disappeared. Examples of these animations can be seen in Fig. 1. As the experiment aimed for realism, the efficacies of gunshots and bandages were randomly varied, such that on average, it would take around $10 \mathrm{~s}$ to complete the task. The actual mean trial duration was $10.5 \mathrm{~s}, \mathrm{SD}=0.85 \mathrm{~s}$; total task duration mean was 26 $\min , \mathrm{SD}=2 \mathrm{~min} 38 \mathrm{~s}$ corresponding to 120 trials (20 bandaging nonhumans; 20 shooting nonhumans; 20 bandaging males; 20 shooting males; 20 bandaging females; 20 shooting females). To avoid order effects, trials from different conditions were interleaved pseudo randomly in 20 blocks containing 1 trial from each condition.

During the interactions with the target, the target itself was programmed to move toward the participant. The nonhumans behaved aggressively to the participant, leaping forward and attacking with blades, while the humans simply walked slowly toward the participant. Once the target was completely healed or killed, they faded from the environment and the door to the next room opened. Log files were analyzed to confirm that subjects had completed the tasks and to derive onset times and epoch durations for the imaging analysis.

\section{Image acquisition and data analysis}

Data were acquired using a 2 T Siemens Magnetom VISION whole body MRI system (Siemens GmbH, Erlangen, Germany) equipped with a head volume coil. A structural MRI scan using a standard three-dimensional $\mathrm{T} 1$ weighted sequence was acquired from each participant. Functional T2*-weighted images were obtained using echo-planar imaging [echo time $(\mathrm{TE})=40 \mathrm{~ms}$ ]. Volumes were acquired continuously for the whole head: 32 slices, each $3 \mathrm{~mm}$ thick, $3.17 \mathrm{~s}$ per volume. Image analysis was performed using SPM99 (Wellcome Department of Imaging Neuroscience, London, UK; Friston et al., 1995) in a standard manner. Briefly, the functional images were realigned (with each other) and coregistered with the structural scan. The images were normalized to a standard template (Montreal Neurological Institute) and spatially smoothed with a Gaussian filter $(6 \mathrm{~mm}$ 

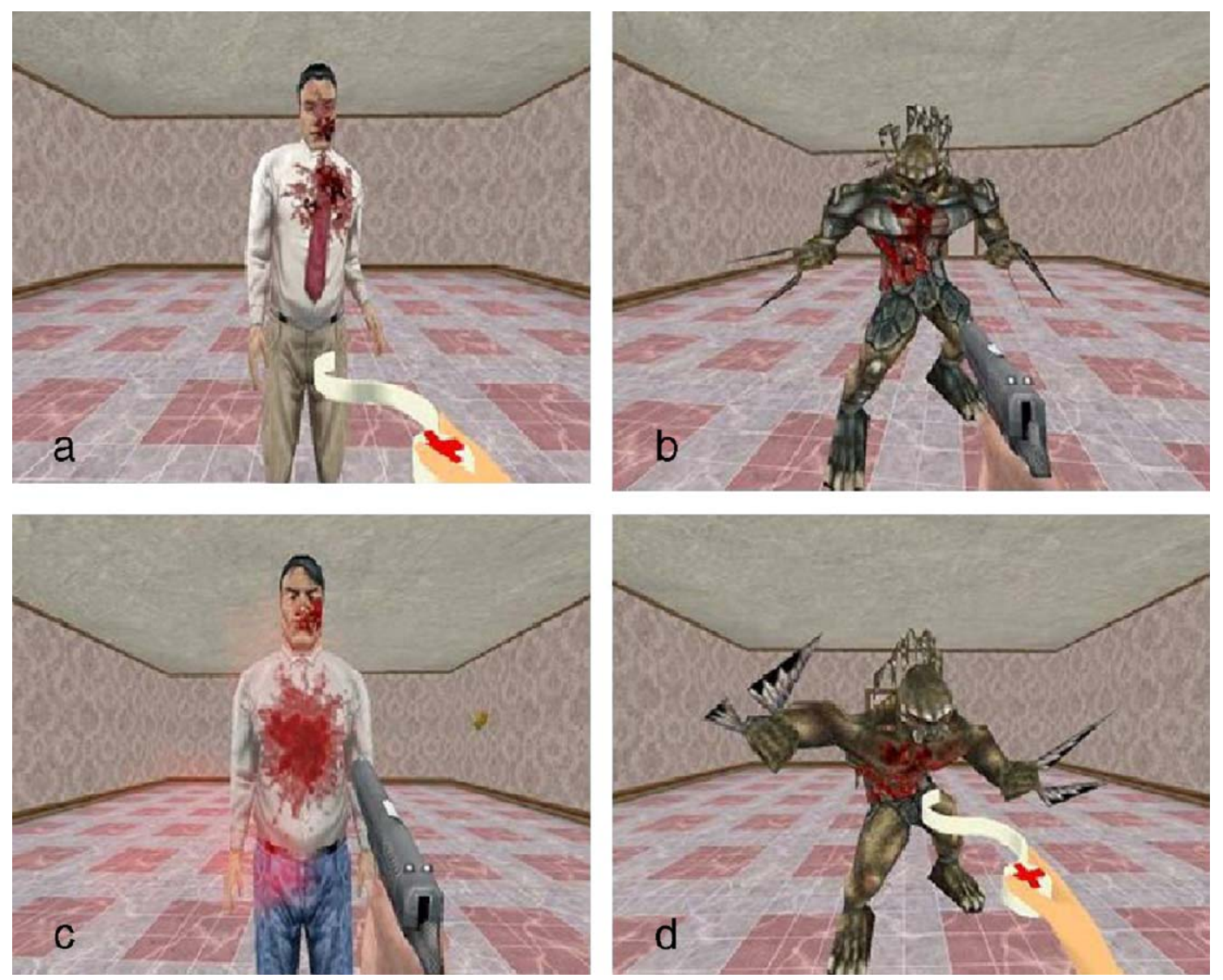

Fig. 1. Stimuli from each trial type showing the two types of appropriate and inappropriate behavior within the context of a video game: (a) appropriate compassion, bandaging a wounded human; (b) appropriate violence, shooting a nonhuman assailant; (c) inappropriate violence, shooting a wounded human; (d) inappropriate compassion, bandaging a nonhuman assailant.

full width half maximum). For each individual, the fMRI timeseries was high pass filtered and modeled as the weighted sum of regressors corresponding to effects of interest. All conditions were modeled as variable length epochs, the duration of each epoch depending on the time taken to complete the interaction with the target (mean $10.5 \mathrm{~s}$ ). The timings were derived from logs produced by the scanner and the VR engine, the latter sending signals to the former via the computer's parallel port to signal the onset and offset of a trial. These regressors were convolved with the canonical hemodynamic response function. Finally, the model also included regressors based on estimates of head-movement obtained from the realignment procedure (to account for any second order effect of such movement remaining after realignment). Initial analysis found no interesting differences between male and female figures, so these trials were collapsed together. Thus, the model consisted of 4 regressors of interest corresponding to bandaging humans, shooting humans, bandaging nonhumans, and shooting nonhumans. The experiment can thus be considered as a $2 \times 2$ factorial design: [shoot, bandage] $\times$ [passive human, nonhuman assailant].

The weights for the best fitting model (or 'parameter estimates') were found, and subjected to a random effects analysis. That is, for each voxel in the brain, single sample $t$ tests were used to determine whether the estimated contrast of parameter estimates between conditions (e.g., bandaging humans minus shooting humans) was significantly different from zero. The activation loci we report exceed a threshold for statistical significance of $P<0.001$ (uncorrected for multiple comparisons) and an extent of 5 or more voxels. To establish which activations were common to the two appropriate behavior conditions, a conjunction analysis (Price and Friston, 1997) was performed (using SPM2 to allow us to incorporate the more conservative conjunction-null assumption; Brett et al., 2004). Results of this analysis are shown in Fig. 4. In addition, we calculated percent signal change to examine the patterns of activation in the main effects for each condition compared to the baseline level of activation (which corresponds to all activation during the experiment not accounted for by the experimental epochs). Finally, the ventromedial frontal activations were superimposed on a mean functional image to confirm that they were not affected by signal loss which can affect inferior frontal regions (see Fig. 5a).

\section{Results}

Participants were verbally debriefed after scanning and asked to rate each condition for how disturbing they found it. Ratings were on a scale of 1 (not disturbing) to 7 (very disturbing) and we found a significant interaction between the target (assailant/ casualty) and object (gun/bandage) factors $(F[1,12]=22.5, P<$ 0.001; mean scores and standard deviations were: shoot-assailant 2.46(1.51); heal-assailant 3.23(1.48); shoot-casualty 4.07(1.26); heal-casualty $2.31(1.15)$ ). We took high ratings to be an indirect reflection of engagement in a behaviour that was felt to be inappropriate, consistent with informal comments made during debriefing. 

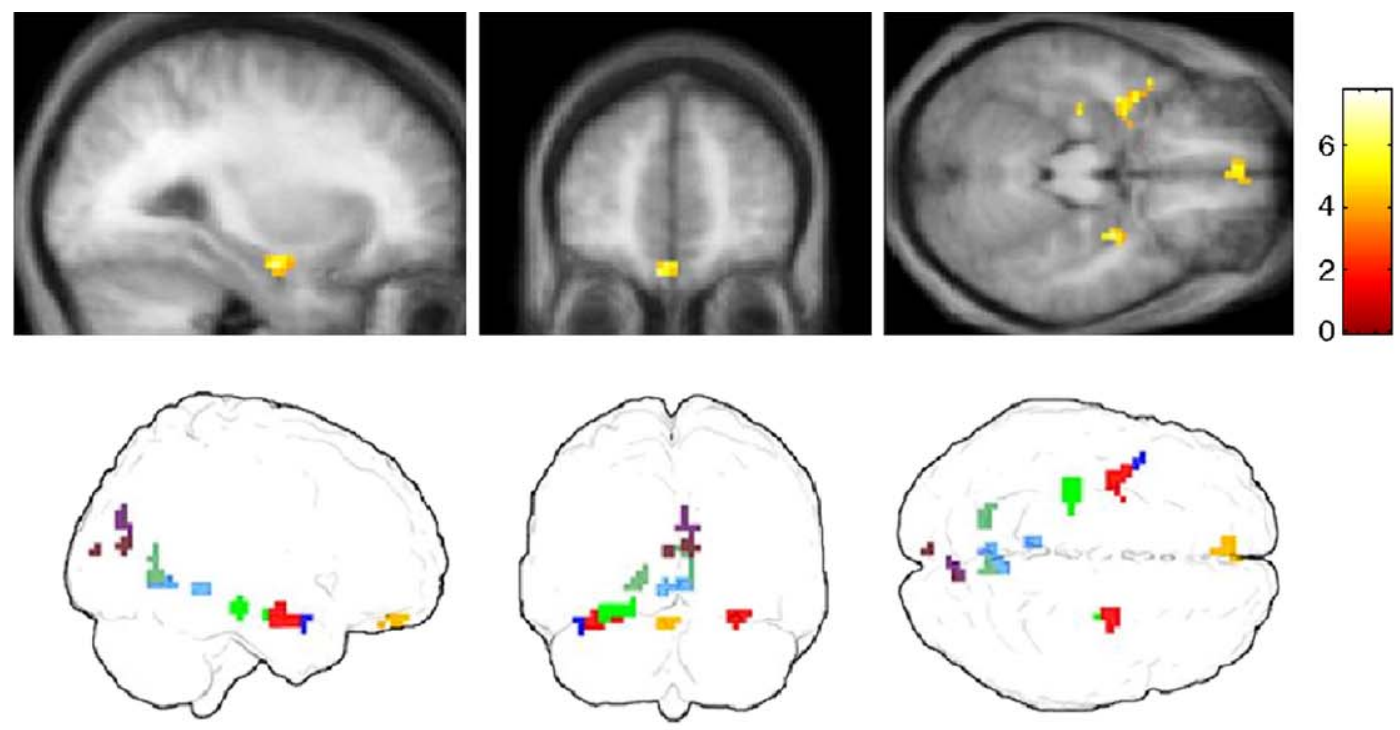

\begin{tabular}{|c|c|c|c|c|}
\hline Anatomical location of peak & Brodmann area & $(x, y, z)$ & $z$ & Exten \\
\hline L Amygdala & & $(-36-6-21)$ & 3.78 & 32 \\
\hline L Anterior Insula & BA 38 & $(-363-21)$ & 3.73 & - \\
\hline R Hippocampus & & $(30-9-21)$ & 4.1 & 17 \\
\hline R Amygdala & & $(33-6-18)$ & 3.87 & - \\
\hline L Ventromedial prefrontal cortex & BA 11 & $(-648-24)$ & 4.06 & 15 \\
\hline L Hippocampus & & $(-30-24-18)$ & 4.45 & 25 \\
\hline R Cuneus & BA 18 & $(9-7812)$ & 3.63 & 21 \\
\hline R Precuneus & BA 31 & $(12-7521)$ & 3.29 & - \\
\hline R Cuneus & BA 18 & $(0-909)$ & 3.53 & 5 \\
\hline L Lingual Gyrus & BA $18 / 19$ & $(-18-63-6)$ & 3.53 & 15 \\
\hline R Cerebellum & & $(6-57-6)$ & 4.15 & 30 \\
\hline R Lingual Gyrus & BA 17 & $\left(\begin{array}{lll}6 & -63 & 12\end{array}\right)$ & 3.6 & - \\
\hline L Cerebellum & & $(-6-45-9)$ & 3.57 & 9 \\
\hline
\end{tabular}

Fig. 2. Frontal and medial activations of interest associated with appropriate violent and compassionate behavior versus matched inappropriate behavior (sagittal, coronal, and horizontal sections through $x=30, y=49, z=-22$ in Talairach space shown on the participants' mean structural image). The glass brains show projections of the data into orthogonal views with activations colored as per the table. Regions with no extent denote subpeaks of contiguous activations: left anterior insula with amygdala; right amygdala with hippocampus; right precuneus with cuneus; right lingual gyrus with cerebellum. See Materials and methods for details.

We compared the neural response to shooting assailants and healing casualties (the appropriate behavior conditions within our video game-like context) with the neural response to matched conditions of healing assailants and shooting casualties (inappropriate behaviors within our context). Significant activations in this comparison (summarized in Fig. 2, see Materials and methods) included ventromedial prefrontal cortex, amygdala and anterior hippocampus bilaterally, and left anteroventral insula. Fig. 2 shows the prefrontal and limbic areas of interest.

To examine the regions of interest in more detail, percent signal change relative to baseline was calculated for each participant in each condition, see Fig. 3 and Materials and methods. This showed a highly consistent pattern of neural responses across areas in response to the appropriate behavior conditions relative to the conditions where the same behaviors were not appropriate.

To verify that a common circuit is involved in both appropriate violence (shooting assailants) and appropriate compassion (healing casualties), we calculated the conjunction image for these two main effects, displaying voxels which were activated in both, see Materials and methods. The results of this analysis are illustrated in Fig. 4. In summary, the predicted network of ventromedial prefrontal cortex and amygdala is commonly activated in both these conditions. A number of other areas of activation are also common to the two, most prominently an activation of the right insula (dorsal and posterior to the contralateral activation in the appropriate versus inappropriate contrast, see Fig. 4). However, since these activations do not appear in the main contrast (Fig. 2), they must also be present to some extent in the inappropriate conditions.

We also examined whether the proposed network for contextappropriate action might reflect other functional divisions of our conditions, consistent with other hypotheses relating to the regions activated. The contrast of inappropriate versus appropriate responding might be predicted to show inferior frontal activation 
Left hippocampus

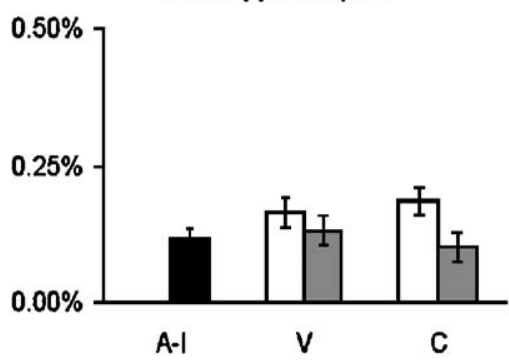

Left amygdala

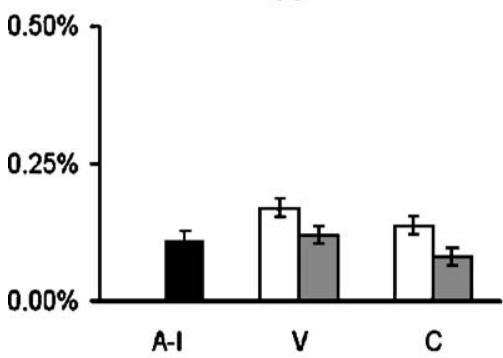

Ventromedial pfc

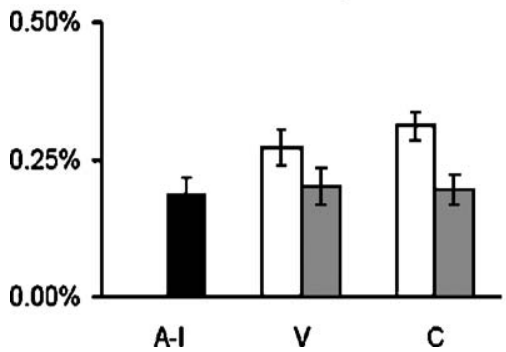

Right hippocampus

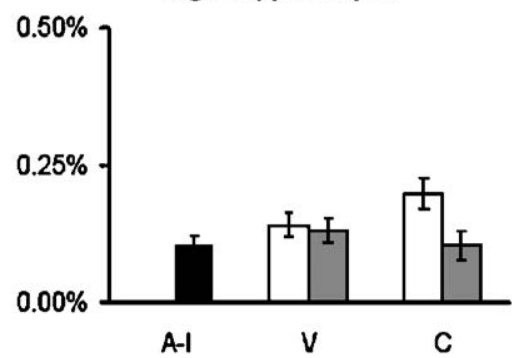

Right amygdala

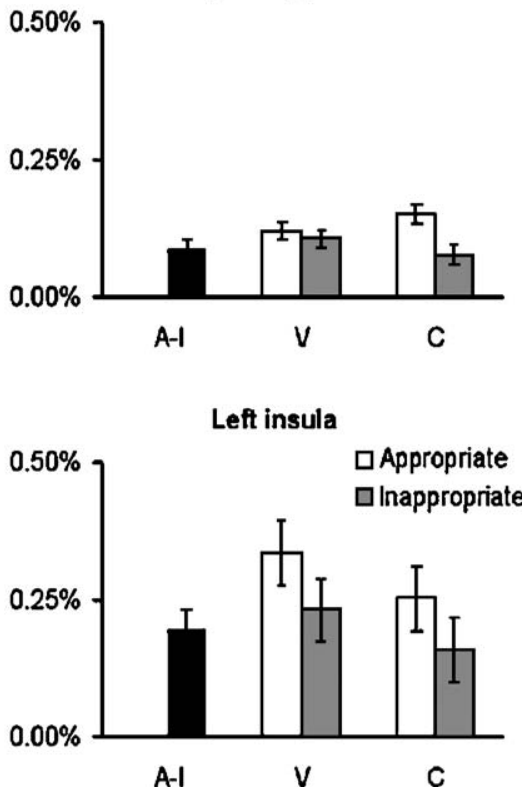

Fig. 3. Mean percent signal change relative to the baseline for each condition. The first column (A-I) shows the sum of appropriate minus inappropriate values, i.e., (shooting assailants + bandaging casualties) - (shooting humans + bandaging casualties). Error bars are 1 SEM. The remaining columns show how this breaks down into violent ( $\mathrm{V}$ - shooting) and compassionate (C-bandaging) behaviors. Error bars for these are 1 SEM of the differences between conditions.

under a hypothesized role in over-ruling natural or pre-potent responses or response inhibition (Aron et al., 2004; Garavan et al., 1999). In fact, this contrast showed only bilateral activation of the supramarginal gyrus (see Fig. 5b). The contrast of violent responses (shooting) with compassionate responses (bandaging) might be predicted to show amygdala and ventromedial prefrontal activation under a hypothesized role in aggression (Anderson et al., 1999; Grafman et al., 1996; Gregg and Siegel, 2001; Panksepp, 1998). Again, we found little activation in this contrast, other than extensive bilateral activation of occipital visual areas (see Fig. 5c). Finally, the contrast of responses made to threatening versus passive stimuli might be predicted to show amygdala involvement (Amaral, 2002; LeDoux, 1998; Whalen et al., 2001). However, activation in this contrast was restricted to bilateral occipital and parietal areas and a single locus in left motor cortex (see Fig. 5d).

\section{Discussion}

In this study, we examined the neural correlates of the expression of appropriate behaviors within the context of a video game. We observed significant activation of orbitofrontal/ventro- medial prefrontal cortex, bilateral amygdala and hippocampus, and left anterior ventral insula when the participant was engaged in appropriate violent or compassionate behavior relative to carefully matched conditions in which the same behaviors and stimuli were inappropriately combined. These results support previous neuropsychological studies that have suggested a role for ventromedial frontal cortex and the amygdala in socially appropriate behavior (Blair, 2004; Damasio, 1994; Grafman et al., 1996). Our results extend these findings to healthy volunteers, and suggest a common neural system, which includes ventromedial cortex and the amygdala, and provides a signal to guide the expression of behavior appropriate to the social or societal context, whether this behavior is violent or compassionate (or alternatively, given the absence of choice in the paradigm, reflects the cognitive or affective consequences of engaging in normative behaviour). This model is broadly consistent with a previous characterization of the role of the amygdala in social cognition (Emery and Amaral, 2000): that, by virtue of somatic sensory input via the insula and social contextual information from orbitofrontal cortex, the amygdala performs a socio-affective evaluation of the salience of sensory stimuli. By virtue of its many connections with cortical and subcortical regions, it can then influence the expression of contextually appropriate responding to social signals. 

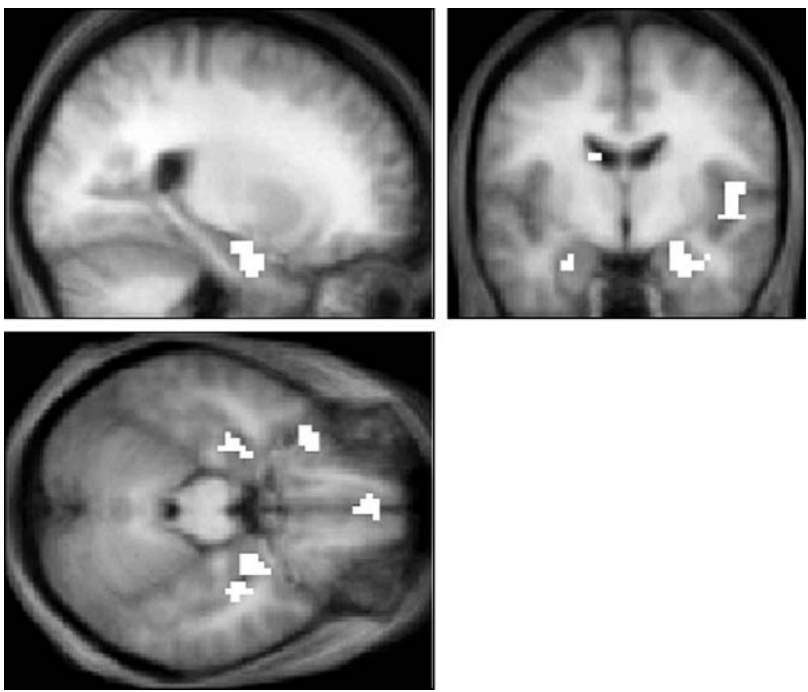

Fig. 4. Sections through the participants' mean structural image showing the conjunction of main-effects for the appropriate behaviors (shooting assailants and bandaging casualties); note the presence of amygdala (peaks at coordinate $-24-6-27, z=4.29$ and $27-3-27, z=4.71$ ), and orbitofrontal cortex (peak at $048-24, z=4.8$ ) showing that these are active in both violent and nonviolent behavior. The activations are significant at $P<0.001$, uncorrected for multiple comparisons, see Materials and methods for details.

It is important to note that the involvement of the amygdalaventromedial prefrontal circuit in context-appropriate behavior was, if anything, more pronounced during compassionate than violent behavior. That is, this circuit is specifically involved in showing compassion to a conspecific, as well as in showing aggression to the violent assailant. These results are consistent with a body of work suggesting a broader role for the amygdala than solely processing aversive or threatening stimuli (Everitt et al., 2000; Hamann and Mao, 2002). While previous studies have implicated both the amygdala and ventromedial prefrontal cortex in aggression (Anderson et al., 1999; Grafman et al., 1996; Gregg and Siegel, 2001; Panksepp, 1998), there has been relatively little work concerning the structures involved in prosocial or compassionate behavior (but see Singer et al., 2004 and below). A study of the areas associated with reward processing during mutual cooperation (Rilling et al., 2002) identified only the medial frontal cortex of the areas identified in our study.

Beyond the amygdala-ventromedial prefrontal system, appropriate violent and compassionate behavior was also associated with anterior ventral insula activity. The anterior insula is activated in studies of pain (Ploghaus et al., 2001) and anticipation (Critchley et al., 2001) and empathy for pain felt by others (Singer et al., 2004) and receives a rich visceroceptive input via the spino-thalamo-cortical pathway (Craig, 2003). Thus, one possibility is that this region represents interoceptive states of arousal. However, we have no reason to believe that there is greater arousal in the appropriate responding conditions as opposed to the control conditions. An alternative possibility is that the insula provides the neural substrate for a biasing signal on behavior that facilitates context appropriate responses. Such a biasing signal might involve so called 'somatic markers' for empathy or threat (Damasio, 1994).

Context-appropriate responding was also associated with bilateral hippocampal activity. One possible explanation for the observed activity is that the socially appropriate conditions represent emotionally charged personally-relevant autobiographical events which tend to activate the hippocampus (Burgess et al., 2001; Dolcos et al., 2004; Maguire et al., 2000; Maguire and Mummery, 1999). That is, the appropriate conditions may have been more personally relevant in the sense of having more in common with memories of previous experiences (albeit hopefully less extreme) in which their responses would likely have been appropriate rather than inappropriate. Direct projections from medial prefrontal cortex to the CA1 field of the hippocampus (Insausti and Munoz, 2001; Suzuki and Amaral, 1990) may support this processing. In addition, a relatively large neuroimaging and neuropsychological literature exists identifying the amygdala's role in enhancing the encoding and consolidation of emotional memoranda, via its interaction with the hippocampus (Cahill, 2000; Hamann, 2001; Hamann et al., 1999; Maratos et al., 2001; Richardson et al., 2004). Thus, the strong hippocampal
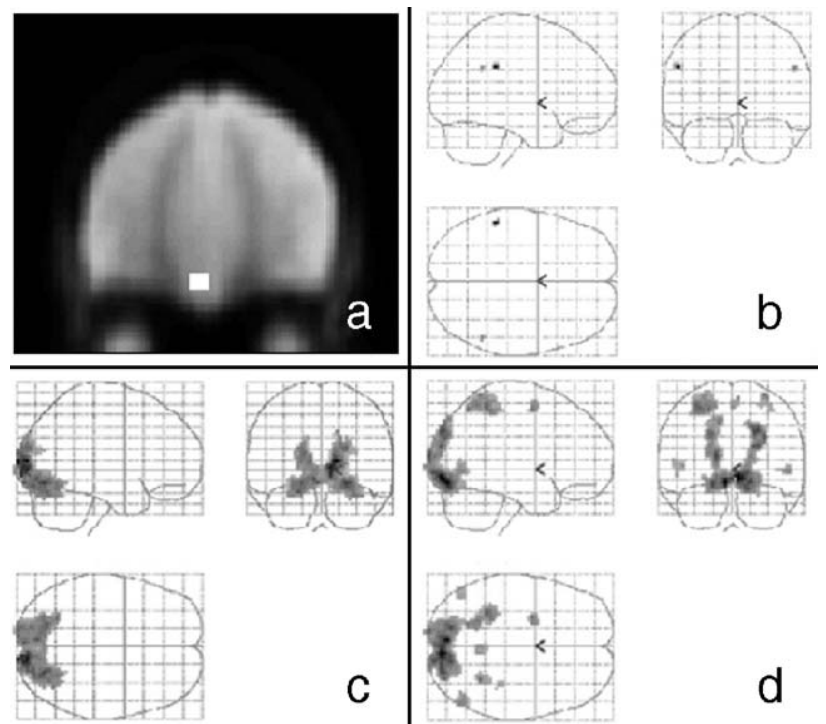

b

Fig. 5. (a) Coronal section through the participants' mean functional image showing the frontal activation, illustrating that the region was not subject to signal loss commonly found in the anterior inferior parts of the brain. (b) Activations in the contrast of inappropriate versus appropriate behavior shown as orthogonal projections through a 'glass brain'. Peaks were found in left and right Supramarginal Gyrus, BA40 $(51-5130 ; z=3.52)$ and $(-54-3933 ; z=4.16)$. (c) Activations in the contrast of violent versus compassionate behavior. The activations are restricted to early visual regions with peaks as follows: right Middle Occipital Gyrus, BA18 (15 -90 $9, z=5.63)$, BA19 (33 -78 18, $z=3.48)$; bilateral Lingual Gyrus, BA17 (12 $-900, z=5.32)$, BA18 $(-6-81-9, z=4.64)$; right Fusiform Gyrus, BA19 $(27-63-12, z=4.21)$; bilateral Cuneus, BA18 $(-15-9915, z=$ $4.43)$ and $(12-999, z=5.33)$. (d) Activations in the contrast of behavior in a threatening versus nonthreatening environment. Peaks were found in visual and visuomotor regions as follows: bilateral Inferior Parietal Lobule, BA40 ( $-30-4557, z=4.55$ and $(27-4557, z=3.46)$; left Inferior Temporal Gyrus, BA19 $(-51-72-6, z=3.48)$; bilateral Lingual Gyrus, BA18 $(-6-81-12, z=5.56)$ and $(6-87-9, z=5.68)$; bilateral Middle Temporal Gyrus, BA37 $(-45-693, z=3.64)$ and $(-54-696, z=3.4)$; left Precentral Gyrus, BA4/6 ( $-27-354, z=4.36)$; right Postcentral Gyrus, BA3 (36 -33 57, $z=3.44)$, BA5 (30 -51 69, $z=4.41)$; left Precuneus, BA7 ( $0-5157, z=3.9)$; left Superior Parietal Lobule, BA7 $(-21-6354, z=4.34)$. The motor activation may reflect the increased difficulty of targeting the more active nonhuman assailants than the passive humans. 
response might also be a consequence of the engagement of the participant's emotional response and amygdala activation. Although memory was not tested in the current study, this explanation would predict that this hippocampal activity would be reflected in improved recall for these events (Cahill, 2000; Hamann, 2001; Hamann et al., 1999).

In conclusion, our results suggest that the expression of context-appropriate behavior in healthy participants is guided by a common neural system including the amygdala and ventromedial prefrontal cortex. These data support suggestions that dysfunction in this system underlies the presentation of inappropriate social behavior in some individuals (Blair and Cipolotti, 2000; Damasio, 1994; Davidson et al., 2000; Grafman et al., 1996). The paradigm presented here provides a way to begin to investigate the neural bases of socially appropriate behavior, how they fail in conditions such as psychopathy, and how this system is affected by manipulation of the (virtual) contexts encountered, or of the prior experience or pharmacological state of the subject.

\section{Acknowledgments}

We would like to thank Uta Frith for helpful discussions during the preparation of the manuscript and Tom Hartley for assistance in preparation of figures. This work was supported by the Medical Research Council and the Wellcome Trust, UK.

\section{References}

Amaral, D.G., 2002. The primate amygdala and the neurobiology of social behavior: implications for understanding social anxiety. Biol. Psychiatry $51,11-17$.

Amaral, D.G., Price, J.L., 1984. Amygdalo-cortical projections in the monkey (Macaca fascicularis). J. Comp. Neurol. 230, 465-496.

Anderson, S.W., Bechara, A., Damasio, H., Tranel, D., Damasio, A.R., 1999. Impairment of social and moral behaviour related to early damage in human prefrontal cortex. Nat. Neurosci. 2, 1032-1037.

Aron, A.R., Robbins, T.W., Poldrack, R.A., 2004. Inhibition and the right inferior frontal cortex. Trends Cogn. Sci. 8, 170-177.

Berthoz, S., Armony, J., Blair, R.J.R., Dolan, R., 2002. Neural correlates of violation of social norms and embarrassment. Brain 125, 1696-1708.

Blair, R.J.R., 2003. Neurobiological basis of psychopathy. Br. J. Psychiatry $182,5-7$.

Blair, R.J., 2004. The roles of orbital frontal cortex in the modulation of antisocial behavior. Brain Cogn. 55, 198-208.

Blair, R.J.R., Cipolotti, L., 2000. Impaired social response reversal: a case of "acquired sociopathy". Brain 123, 1122-1141.

Brett, M., Nichols, T., Andersson, A., Wager, T., Poline, J.B., 2004. When is a conjunction not a conjunction? (Poster Presented at the 10th International Conference on Functional Mapping of the Human Brain). NeuroImage, 22.

Burgess, N., Maguire, E.A., Spiers, H.J., O’Keefe, J., 2001. A temporoparietal and prefrontal network for retrieving the spatial context of lifelike events. NeuroImage 14, 439-453.

Cahill, L., 2000. Neurobiological mechanisms of emotionally influenced, long-term memory. Prog. Brain Res. 126, 29-37.

Craig, A.D., 2003. Interoception: the sense of the physiological condition of the body. Curr.Opin. Neurobiol. 13, 500-505.

Critchley, H.D., Mathias, C.J., Dolan, R.J., 2001. Neural activity in the human brain relating to uncertainty and arousal during anticipation. Neuron 29, 537-545.
Damasio, A.R., 1994. Descartes' Error: Emotion, Rationality and the Human Brain. Putnam (Grosset Books), New York.

Davidson, R.J., Putnam, K.M., Larson, C.L., 2000. Dysfunction in the neural circuitry of emotion regulation-A possible prelude to violence. Science 289, 591-594.

Dolcos, F., LaBar, K.S., Cabeza, R., 2004. Interaction between the amygdala and the medial temporal lobe memory system predicts better memory for emotional events. Neuron 42, 855-863.

Emery, N.J., Amaral, D.G., 2000. The role of the amygdala in primate social cognition. In: Lane, R.D., Nadel, L. (Eds.), Cognitive Neuroscience of Emotion. Oxford Univ. Press, New York, pp. 156-191.

Everitt, B.J., Cardinal, R.N., Hall, J., Parkinson, J.A., Robbins, T.W., 2000. Differential involvement of amygdala subsystems in appetitive conditioning and drug addiction. In: Aggleton, J.P. (Ed.), The Amygdala: A Functional Analysis. Oxford Univ. Press, Oxford, pp. 289-310.

Friston, K.J., Holmes, A.P., Worsley, K.J., Poline, J.-P., Frith, C.D., Frackowiak, R.S., 1995. Statistical parametric maps in functional imaging: a general linear approach. Hum. Brain Mapp. 2, 189-210.

Garavan, H., Ross, T.J., Stein, E.A., 1999. Right hemispheric dominance of inhibitory control: an event-related functional MRI study. Proc. Natl. Acad. Sci. U. S. A. 96, 8301-8306.

Grafman, J., Schwab, K., Warden, D., Pridgen, B.S., Brown, H.R., 1996. Frontal lobe injuries, violence, and aggression: a report of the Vietnam head injury study. Neurology 46, 1231-1238.

Gregg, T.R., Siegel, A., 2001. Brain structures and neurotransmitters regulating aggression in cats: implications for human aggression. Prog. Neuro-Psychopharmacol. Biol. Psychiatry 25, 91-140.

Hamann, S., 2001. Cognitive and neural mechanisms of emotional memory. Trends Cogn. Sci. 5, 394-400

Hamann, S., Mao, H., 2002. Positive and negative emotional verbal stimuli elicit activity in the left amygdala. NeuroReport 13, 15-19.

Hamann, S.B., Ely, T.D., Grafton, S.T., Kilts, C.D., 1999. Amygdala activity related to enhanced memory for pleasant and aversive stimuli. Nat. Neurosci. 2, 289-293.

Hare, R.D., 1991. The Hare Psychopathy Checklist-Revised. Multi-Health Systems, Toronto, Ontario.

Insausti, R., Munoz, M., 2001. Cortical projections of the non-entorhinal hippocampal formation in the cynomolgus monkey (Macaca fascicularis). Eur. J. Neurosci. 14, 435-451.

Kiehl, K.A., Smith, A.M., Hare, R.D., Mendrek, A., Forster, B.B., Brink, J., Liddle, P.F., 2001. Limbic abnormalities in affective processing by criminal psychopaths as revealed by functional magnetic resonance imaging. Biol. Psychiatry 50, 677-684.

LeDoux, J., 1998. The Emotional Brain. Weidenfeld and Nicolson, New York.

Maguire, E.A., Mummery, C.J., 1999. Differential modulation of a common memory retrieval network revealed by positron emission tomography. Hippocampus 9, 54-61.

Maguire, E.A., Mummery, C.J., Buchel, C., 2000. Patterns of hippocampal-cortical interaction dissociate temporal lobe memory subsystems. Hippocampus 10, $475-482$.

Maratos, E.J., Dolan, R.J., Morris, J.S., Henson, R.N., Rugg, M.D., 2001. Neural activity associated with episodic memory for emotional context. Neuropsychologia 39, 910-920.

Panksepp, J., 1998. Affective Neuroscience: The Foundations of Human and Animal Emotions. Oxford Univ. Press, New York.

Pietrini, P., Guazzelli, M., Basso, G., Jaffe, K., Grafman, J., 2000. Neural correlates of imaginal aggressive behavior assessed by positron emission tomography in healthy subjects. Am. J. Psychiatry 157, $1772-1781$.

Ploghaus, A., Narain, C., Beckmann, C.F., Clare, S., Bantick, S., Wise, R., Matthews, P.M., Rawlins, J.N., Tracey, I., 2001. Exacerbation of pain by anxiety is associated with activity in a hippocampal network. J. Neurosci. 21, 9896-9903.

Price, C.J., Friston, K.J., 1997. Cognitive conjunction: a new approach to brain activation experiments. NeuroImage 5, 261-270.

Richardson, M.P., Strange, B.A., Dolan, R.J., 2004. Encoding of emotional 
memories depends on amygdala and hippocampus and their interactions. Nat. Neurosci. 7, 278-285.

Rilling, J., Gutman, D., Zeh, T., Pagnoni, G., Berns, G., Kilts, C., 2002. A neural basis for social cooperation. Neuron 35, 395-405.

Singer, T., Seymour, B., O’Doherty, J., Kaube, H., Dolan, R.J., Frith, C.D., 2004. Empathy for pain involves the affective but not sensory components of pain. Science 303, 1157-1162.
Suzuki, W.A., Amaral, D.G., 1990. Cortical inputs to the CA1 field of the monkey hippocampus originate from the perirhinal and parahippocampal cortex but not from area TE. Neurosci. Lett. 115, $43-48$.

Whalen, P.J., Shin, L.M., McInerney, S.C., Fischer, H., Wright, C.I., Rauch, S.L., 2001. A functional MRI study of human amygdala responses to facial expressions of fear versus anger. Emotion 1, 70-83. 